\title{
A Distant node Based Multicast Routing protocol for Sparse area vehicle to vehicle communication
}

\author{
Dibakar Chakraborty \\ (Department Of Computer Science and Engineering, National Institute of Technology, Agartala)
}

\begin{abstract}
Inter-vehicular communication(IVC) is one of the most advanced research areas for last few years. Traditional Mobile-adhoc-network(MANET) protocols are proved to be not that much effective in Vehicularadhoc-network(VANET). Moreover the protocols for dense areas are not suitable for partially connected areas. In this paper a Distance Node Based Multicast Routing (DBMR) protocol for sparse areas with a small overhead of incorporating multicast group selection is introduced with some small modification in the existing Border node Based Routing $(B B R)$ protocol.The performance of DBMR is evaluated in NS-2 simulator with VanetMobiSim based mobility model. The simulation results verify that the multicast behavior of the proposed $D B M R$ exploits a distinct bandwidth utilization over the flooding behavior of BBR.
\end{abstract}

Keywords: Broadcast, Distant nodes, IVC, Multicast, Routing protocols.

\section{INTRODUCTION}

The recent advance in wireless communication technology has made inter vehicle communication and vehicle to road side communication feasible in real environment. Vehicular Ad hoc network(VANET)[1] can be thought of as a special case of Mobile ad hoc network(MANET) but with some unique features in it. Recognizing the importance of VANET the Federal Communications Commission (FCC) has allocated 75 $\mathrm{MHz}$ spectrum at $5.9 \mathrm{GHz}$ for dedicated short range communications(DSRC)[3].

VANET is characterized by rapidly moving nodes and the incessantly changing network topologies. However the nodes move in predefined routes and their trails aren't so much complicated. VANET can offer the platform for the applications like traffic management, commercial applications and sharing information between cars.

However, it is evident that the traditional MANET protocols[9] can't be directly implemented for VANET without any modifications. As the topology is changing rapidly it is very hard to maintain the routes and frequent disconnections also occur because of high mobility of vehicles. The routing protocols available for VANET are mostly made suitable for urban and sub-urban areas assuming high vehicle density. The scenario of rural and sparse areas are different the issues like low node-density, large inter-vehicle spacing and terrain effects make it harder to maintain the routes. Moreover the unavailability of the fixed infrastructures enhances the difficulty. Sparse area VANET can be thought of as a partially connected MANET whre the node density is low and the node mobility is high.

Based on the above discussed constraints in the sparse areas a Distant node Based Multicast Routing protocol is proposed in this paper and some of the existing VANET protocols are studied. The Border node based Routing protocol approach which has some of the attributes of the epidemic protocol is also explored.

The rest of the paper is structured as follows: Section II represents the related research works. In Section III the DBMR protocol is discussed in detail. Section IV includes the simulation results. The final section concludes the paper.

\section{RELATED WORKS}

\subsection{Border node Based Routing protocol}

BBR protocol is based on some approaches of the epidemic routing protocol. It is based on certain assumptions like unavailability of location information, message oriented communication without any fixed infrastructure 
The existing BBR protocol constitutes of two functional units namely neighbor discovery algorithm and border node selection algorithm. The neighbor discovery algorithm collects current one hop neighbor information by sending periodic 'hello' messages and performs routing actions in the network layer without consideration of the underlying MAC layer technology used.

Border nodes are selected per broadcast event, which stores the broadcast information and forwards the data packets. The border node selection is based on the assumption that the nodes located at the edge of transmission range has a least no. of common neighbors and they will meet more new neighbors than the nodes closer to the current source.

\subsection{V-to-V communication(routing) protocols}

Vehicular ad-hoc networks differ from classical ad-hoc networks by certain characteristics. First, node mobility is restricted by road layouts, traffic rules and other node's movements. Moreover, external factors like weather conditions effect the node movements. With view of the above points traditional ad-hoc routing protocols can't be suitable for VANET routing.

There are three classes of MANET routing protocols; proactive, reactive and position based routing protocols by category.

Naumov et. al[4] analyzed AODV and GPRS[5] and showed that they exhibit poor performance in VANET. AODV incorporates a lot of collisions because of too many broadcast and GPSR leads outdated information in neighbor tables.

The position based routing approaches like GPCR[9], , CAR[10], GSR[7], A-STAR[8] computes the shortest path between sender and receiver based on certain position informations .

Vehicle motion information based protocols like MOVE[11] computes the distance based on speed information. Again, Maxprop[12] uses the probabilistic approach of meeting other vehicles for calculation of forwarding path.

The protocols based on link quality like GPSR-L[15] make routing decision based on life time. ACAR[16] is another one which estimates transmission quality based on node density and traffic.

\section{Distant Node BaSed Multicast Routing (DBMR) Protocol}

The DBMR protocol incorporates both the multicast and unicast behavior. The broadcast messages in the BBR protocol brings undesirable large overhead .The multicast behavior of the DBMR protocol encounters this issue to provides effective bandwidth utilization. The DBMR protocol exhibits unicast behavior when the destination node is within the radio range of the current forwarding node.

The DBMR protocol involves a threshold $\tau$ to select the nodes which will be receiving the multicast data packet. DBMR protocol divides it's task into two distinct phases namely, phase1: Neighbor-Groupt creation and Multicast, phase2:Distant node selection. The DBMR protocol uses only the one-hop neighbor information of the current forwarding node which is collected during the Neighbor-List creation phase. The Distant node selection phase is a heuristic based approach. A Distant node is one which has the responsibility of forwarding the data packets using the store-carry-forward approach.

The assumptions in the DBR protocol are very likely to those in the BBR protocol. The assumptions and the phases of the DBMR protocol are discussed in detail below:

\subsection{Assumptions}

The assumptions of the DBMR protocol are taken into account considering the scenario of the sparse areas. First, location information of the nodes are unavailable. Second, The communication issues are the burden of the ad-hoc network solely. Third, node power is not a limiting factor. Fourth, message passing is the only means of communication. Fifth, all the node are equipped with an omni directional antenna of equal transmission range making the links bi-directional.

\subsection{Phase1: Neighbor-Group creation and Multicast}

Neighbor selection is done through two sub-phases namely: Neighbor-List creation and Multicast group selection. The sub-phases are detailed below:

\subsubsection{Neighbor-List creation:}

The current one-hop neighbor collection is the responsibility of the Neighbor-List creation subphase. The current one-hop neighbor of a particular node forms the neighbor-list set. The neighbor nodes share this list for selection of distant nodes. As the sparse and partially connected areas incorporates indeterministic high mobility, the Neighbor-List is to be updated dynamically. A pro-active approach of sending periodic 'hello' message is undertaken to encounter the above issue. The hello messages are network layer based ; they are sent out by the network layer. It is more convenient to sent the 'hello' messages through the network layer because routing functions can be performed without consideration of the underlying MAC layer technology. 


\subsubsection{Multicast group selection}

The existing Border node Based Routing (BBR) protocol floods the network without considering the relative distance between the nodes, resulting in an inefficient bandwidth utilization;Considering this issue, the DBMR protocol introduces a threshold $\tau$ to classify the current one-hop neighbors which will receive the multicast data packet with respect to the current forwarding(Distant) node. The multicast packet receiving nodes are selected on the basis of transmission time (as transmission time in low node density,light traffic area is directly dependent on physical distance between nodes, other factors are negligible). The 'hello' messages contain the current timestamp before it has been sent out. The current forwarding node receives 'hello' messages from it's current one hop neighbors and computes the transmission time and averages two recent successive transmission times of all the one-hop neighbors and then compares with a threshold $\tau$ for selection of data packet receiving neighbors. A node $\mathrm{k}$ is added to the multicast group of current forwarding node $\mathrm{F}$ if the following condition is satisfied.

: $\quad \mathrm{TT}_{\mathrm{k}}(\mathrm{i}+1)+\mathrm{TT}_{\mathrm{k}}(\mathrm{i}) \geq 2 \tau$, for all $\mathrm{k}$ in the one-hop Neighbor-List of $\mathrm{F}$

Where, $\mathrm{TT}_{\mathrm{k}}(\mathrm{i})$ is the computed transmission time for node $\mathrm{k}$ at the $\mathrm{i}^{\text {th }}$ time instant

$\tau$ is proportional to transmission range of nodes

To optimize the utilization of bandwidth and reduce the broadcast overhead, instead of the broadcast behavior of the BBR protocol, multicasting is adopted in the proposed DBMR protocol.

\subsection{Phase2: Distant node selection}

The Distant nodes are selected per multicast event. A Distant node is responsible for storing received multicast data forwarding to appropriate nodes at appropriate time. The Current forwarding(Distant) node multicasts the received data packets only to the nodes those who are the members of the multicast group.It is the responsibility of a particular node to decide whether it is Distant node or not; the current one-hop neighbor information and the received multicast information are used as selection information.

\subsubsection{Criteria for the Distant node selection procedure}

The Distant node/nodes selection criteria in DBMR is similar to the selection of Border node/nodes in the BBR protocol.The selection of a Distant node is based on minimum common neighbor approach.

The minimum common neighbor approach is undertaken upon the intuitive notion that a Distant node situated at the edge of a transmission range should have a fewer common neighbor or the Distant node/nodes must should have a maximum uncommon neighbor with the current multicast source node as compared to those that are closer to the forwarding node(multicast source node).

3.2.2.Implementation of Distant node selection. The DBMR protocol involves store-carry and forward approach like the delay tolerant network. The original creator or source of a data packet is by default a Distant node. The multicast data packet has the following structure as shown in Table1.

Table1: Multicast data packet format

\begin{tabular}{|l|l|l|l|l|l|l|l|}
\hline $\begin{array}{l}\text { Source } \\
\text { ID }\end{array}$ & $\begin{array}{l}\text { Destination } \\
\text { ID }\end{array}$ & $\begin{array}{l}\text { Common } \\
\text { Neighbor } \\
\#\end{array}$ & $\begin{array}{l}\text { Packet } \\
\text { Id/seq. } \\
\text { No }\end{array}$ & $\begin{array}{l}\text { Packet } \\
\text { Content }\end{array}$ & $\begin{array}{l}\text { Neighbor } \\
\text { List }\end{array}$ & $\begin{array}{l}\text { Multi- } \\
\text { Cast } \\
\text { Group }\end{array}$ & Reserved \\
\hline
\end{tabular}

Three tables are needed to be maintained by a particular node namely- Neighbor-List, selection table, message table. The Neighbor-List contains the one-hop neighbor information. Selection table stores the necessary information for the selection of Distant node/nodes. Message table buffers the data packets with the sequence no(packet id). The message table is searched when a new node comes in contact of a particular node to check whether it is a destination of a data packet or not. Reception of duplicate packets are discarded by checking the packet sequence no(packet id). If a new packet arrives a node will perform appropriate action in a specific condition. The condition wise approaches are discussed below: 


\subsubsection{Condition1}

Condition1.1: When the multicast group is empty then the source(sender/forwarding) node doesn't forward it rather buffers the packet in message table and carries the data packet. The typical road layout condition is presented in Fig.1. Here S (shown in black circle) is the multicast source node and it's current multicast group is empty as all the neighboring nodes are incoming in nature ; hence $\mathrm{S}$ carries the data packet instead of forwarding it.

Condition1.2: On the other hand if there is only one member in the multicast group then it will be automatically selected as a Distant node. A typical scenario is presented in Fig.2 to describe situation discussed in condition 1.2 .S is the multicast source node ,nodes a,b, Dt are the current one-hop neighbors of S. But only the node $\mathrm{Dt}$ (shown in a green circle) is in the multicast group of $\mathrm{S}$ hence it is chosen as a Distant node by default.

\subsubsection{Condition2}

When multiple members present in the multicast group ,then after receiving the multicast data packet each and every node of the multicast group initiates two timers namely $\mathrm{T}_{\text {sel }}$ and $\mathrm{T}_{\mathrm{rm}}$, similar to the timers in the existing BBR protocol.

The timer $\mathrm{T}_{\text {sel }}$ is used to track the time instanced when the Distant node selection procedure will start after the multicast data packets are received by the nodes. The timer $T_{r m}$ is used to decide the re-multicast time.The re-multicast packet format is shown in Table2.

Table2: Re-multicast data packet format

\begin{tabular}{|l|l|l|l|l|l|l|l|}
\hline $\begin{array}{l}\text { Source } \\
\text { ID }\end{array}$ & $\begin{array}{l}\text { Destination } \\
\text { ID }\end{array}$ & $\begin{array}{l}\text { Common } \\
\text { Neighbor } \\
\#\end{array}$ & $\begin{array}{l}\text { Packet } \\
\text { Id/seq. } \\
\text { No }\end{array}$ & $\begin{array}{l}\text { Packet } \\
\text { Con- } \\
\text { tent }\end{array}$ & $\begin{array}{l}\text { Neighbor } \\
\text { List }\end{array}$ & $\begin{array}{l}\text { Multi- } \\
\text { Cast } \\
\text { Group }\end{array}$ & Reserved \\
\hline
\end{tabular}

The two timers $\left(\mathrm{T}_{\text {sel }}, \mathrm{T}_{\mathrm{rm}}\right)$ are similar to the timers $\mathrm{T}_{\max }$ and $\mathrm{T}_{\mathrm{ad}}$ in the BBR protocol; the only difference is the removal of broadcast and introduction of multicast behavior after the expiration of the timer $T_{\mathrm{rm}} \cdot \mathrm{T}_{\mathrm{rm}}$ varies with the position of a particular node in the neighbor-list.

During the $\mathrm{T}_{\text {sel }}$ timer period the multicast packet receiving nodes decide whether to re-multicast or not once if the
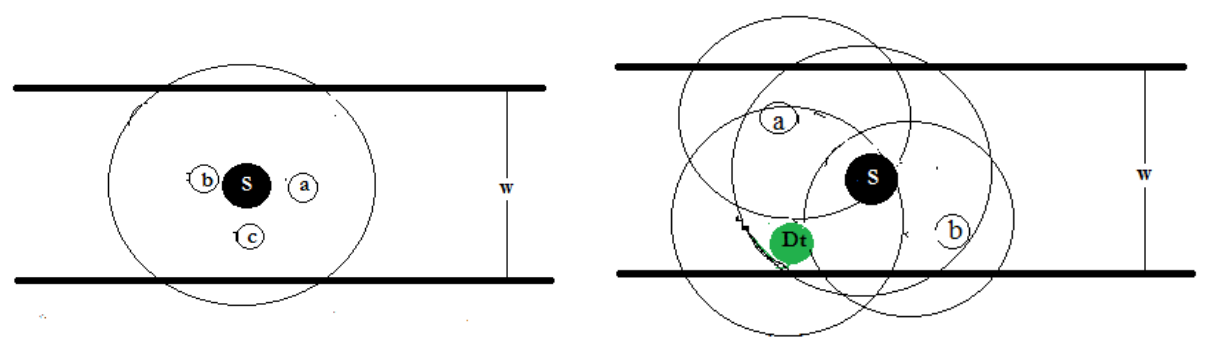

Figure 1. Typical road layout for condition $1.1 \quad$ Figure 2 . Typical road-layout for condition 1.2



Figure 3. Typical road-layout with source $\mathrm{S}$ ( shown as black circle) 
A Distant node Based Multicast Routing protocol for Sparse area vehicle to vehicle communication timer $\mathrm{T}_{\mathrm{rm}}$ of a particular node expires. The $\mathrm{T}_{\text {sel }}$ timer is same for all the nodes. Once $\mathrm{T}_{\text {sel }}$ expires the Distant node selection procedure starts. Condition 2 of Distant node selection procedure is pictorially stated as in Fig.3.

In Fig. 3 the multicast source $s$ is shown as the black node. The motion direction of the vehicles are shown by the arrows. The members of multicast group for node S(shown in black circle) are shown with the filled in green circle in Fig.3. The Distant node selection procedure based on the scenario of Fig.3 is presented in Table 3.

Table3: A typical Distant node selection

\begin{tabular}{|l|l|l|l|l|l|l|}
\hline Node & $\begin{array}{l}\text { Neighbor } \\
\text { list }\end{array}$ & $\begin{array}{l}\text { Multicast } \\
\text { group }\end{array}$ & $\begin{array}{l}\text { Common } \\
\text { Neighbor }\end{array}$ & $\begin{array}{l}\text { Common } \\
\text { Neighbor } \\
\#\end{array}$ & $\begin{array}{l}\text { Action } \\
\text { after } \\
\mathrm{T}_{\mathrm{rm}} \\
\text { expires }\end{array}$ & $\begin{array}{l}\text { Decision } \\
\text { After } \\
\mathrm{T}_{\text {sel }} \\
\text { expires }\end{array}$ \\
\hline $\mathrm{S}$ & $\{\mathrm{b}, \mathrm{d}, \mathrm{f}, \mathrm{e}, \mathrm{i}\}$ & $\{\mathrm{f}, \mathrm{d}, \mathrm{e}\}$ & - & - & - & - \\
\hline $\mathrm{d}$ & $\{\mathrm{s}, \mathrm{c}, \mathrm{b}, \mathrm{f}\}$ & - & $\{\mathrm{b}, \mathrm{f}\}$ & 2 & $\begin{array}{l}\text { Do } \\
\text { nothing }\end{array}$ & - \\
\hline $\mathrm{f}$ & $\{\mathrm{s}, \mathrm{a}, \mathrm{b}\}$ & - & $\{\mathrm{b}\}$ & 1 & $\begin{array}{l}\text { Re- } \\
\text { Multicast }\end{array}$ & $\begin{array}{l}\text { Distant } \\
\text { Node }\end{array}$ \\
\hline $\mathrm{e}$ & $\{\mathrm{s}, \mathrm{i}\}$ & - & $\{\mathrm{i}\}$ & 1 & $\begin{array}{l}\text { Re- } \\
\text { Multicast }\end{array}$ & $\begin{array}{l}\text { Distant } \\
\text { Node }\end{array}$ \\
\hline
\end{tabular}

\section{Performance Evaluation}

In order to evaluate the performance of DBMR, I have used NS-2 simulator version 2.34. A rectangular field of $1000 \mathrm{~m} \times 1000 \mathrm{~m}$ is chosen and simulation time taken is 900 seconds.

\subsection{Simulation setup}

To generate vehicle mobility trace file for VANET in NS-2 the VanetMobiSim is used. VanetMobiSim can generate vehicle mobile topology simulating the operations like vehicle accelarations and decelerations, carto-car and intersection interaction. The MAC protocol used is IEEE 802.11. No. of nodes is 50, and speed of nodes is within the range 0 to $15 \mathrm{~m} / \mathrm{s}$. Initially, all the nodes are uniformly placed in the rectangular area with the average distance $L_{a v}=171.4$ meters. A connectivity parameter $\alpha$ is defined as the ratio between the radio transmission range $(\mathrm{R})$ and the average distance among neighboring nodes $\left(\mathrm{L}_{\mathrm{av}}\right)$, namely $\alpha=\mathrm{R} / \mathrm{L}_{\mathrm{av}}$. The basic parameter values used in simulation are summarized in Table 4.

Table 4. Simulation parameters and values

\begin{tabular}{|l|l|l|l|}
\hline Parameters & Values & Parameters & Values \\
\hline Simulator used & NS-2.34 & Channel type & Wireless Channel \\
\hline Simulation area & $1000 \times 1000 \mathrm{~m}^{2}$ & Queue type & PriQueue \\
\hline No. of vehicles & 50 & Simulation time & 900 secs \\
\hline Vehicle velocity & $0-15 \mathrm{~m} / \mathrm{s}$ & Queue length & 50 \\
\hline MAC protocol & IEEE $802.11 \mathrm{~b}$ & Hello Interval & $1 \mathrm{sec}$ \\
\hline
\end{tabular}

\subsection{Simulation Results}

The performance of DBMR is analyzed in terms of two performance indices- Packet delivery ratio and Average end-to-end delay as a function of radio range.

In Fig.4 it is seen that with the increasing radio range, more precisely with the increase in the connectivity parameter $\alpha$, the packet delivery ratio increases rapidly, but after reaching about $100 \mathrm{~m}$ the ratio remains constant then gradually approaches towards 99\%. In Fig.5 the average end-to-end delay decreases rapidly with increasing radio range. When the radio range is less than the average distance between vehicles i.e $\alpha<1$ then the network is partially connected, causing the large initial delay. The delay decreases gradually when the network become connected( i.e with the increasing $\alpha$ ). 


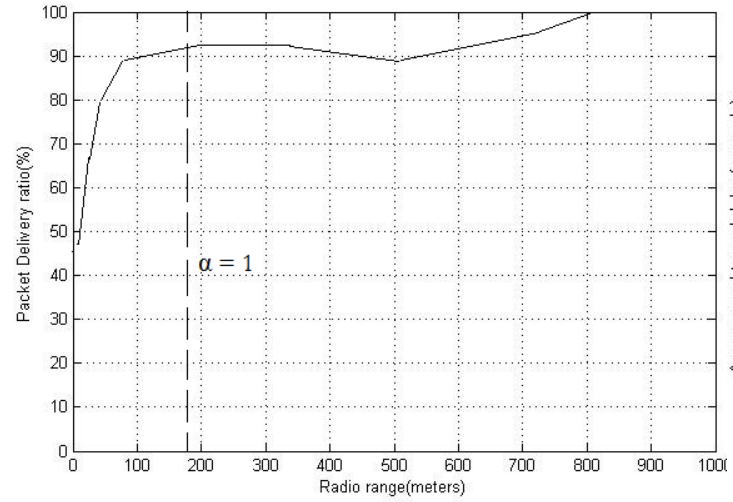

Figure 4. Packet delivery ratio of DBMR

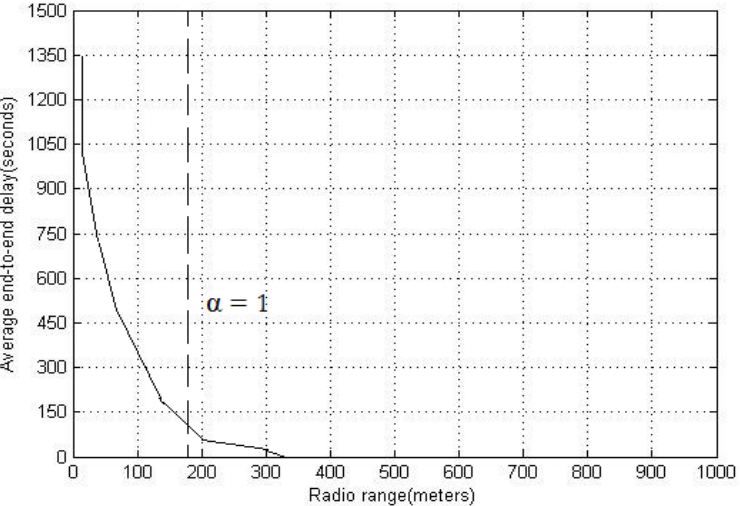

Figure 5. Average end-to-end delay of DBMR

\section{CONCLUSION}

In this paper a Distant node Based Multicast Routing(DBMR) protocol is designed specially for sparse areas. Using NS 2.34 the performance of DBMR is evaluated. This protocol is the direct descendent of the Border node Based Routing (BBR) protocol. It is evident that the position based routing protocols are not that much suitable in rural areas because of terrain effects. Simulation results show the well suitability of DBMR for partially connected networks (with rapid topology change and frequent partitioning) with no position information overhead. The future works include deriving the Distant nodes with more simple methodology.

\section{REFERENCES}

[1] S.Yousefi, M.S. Mousavi, and M. Fathy, “Vehicular Ad hoc Networks (VANETs) : challenges and perspectives," Proc. $6^{\text {th }}$ International Conference on ITS Telecommunications , IEEE Press, Jun 2006, pp.761-766,doi:10.1109/ITST.2006.289012.

[2] M.Zhang and R.S. Wolff,'Border Node-Based Routing Protocols for VANETS in sparse and rural areas",Autonet Workshop,IEEE Globecom, Washington DC,November 2007

[3] Online: DSRC, http://grouper.ieee.org/groups/scc32/dsrc/index.html.

[4] V. Naumov, R. Baumann, and T. Gross, "An evaluation of intervehicle ad hoc networks based on realistic vehicular traces," Proc. $7^{\text {th }}$ ACM International Symposium on Mobile Ad Hoc Networking and Computing, ACM Press, May. 2006, pp. 108-119, doi: 10.1145/1132905.1132918.

[5] B. Karp, and H. Kung, "GPSR: greedy perimeter stateless routing for wireless networks," Proc. 6th Annual International Conference on Mobile Computing and Networking, ACM Press, Aug. 2000, pp. 243- 254, doi: 10.1145/345910.345953.

[6] C. Lochert, et al, "A routing strategy for vehicular ad hoc networks in city environments," Proc. IEEE IV2003 Intelligent Vehicles Symposium, IEEE Press, Jun. 2003, pp. 156-161, doi: 10.1109/IVS.2003.1212901

[7] B. C. Seet, et al, "A-STAR: a mobile Ad Hoc routing strategy for metropolis vehicular communications," Proc. IFIP TC6 Networking, Springer-Verlag Press, May. 2004, pp. 989-999, doi: 10.1007/b97826.

[8] E.Royer and C.Toh,"A review of current Routing Protocols for Ad-Hoc Mobile Wireless Networks",IEEE Personal Communications, Apr. 1999

[9] V. Naumov, and T. Gross, "Connectivity-aware routing (CAR) in vehicular ad-hoc networks," Proc. 26th IEEE International Conference on Computer Communications, IEEE Press, May. 2007, pp. 1919-1927, doi:10.1109/INFCOM.2007.223.

[10] C. Lochert, M. Mauve, H. Fu, and H. Hartenstein, "Geographic routing in city scenarios," ACM SIGMOBILE Mobile Computing and Communications Review, vol. 9, no. 1, Jan. 2005, pp. 69-72, doi:10.1145/1055959.1055970.

[11] J. LeBrun, C.-N. Chuah, D. Ghosal, and M. Zhang, "Knowledgebased opportunistic forwarding in vehicular wireless ad hoc networks," Proc. 61st IEEE Vehicular Technology Conference, IEEE Press, May. 2005, vol. 61, no.4 , pp. 2289-2293, doi: 10.1109/VETECS.2005.1543743.

[12] J. Burgess, B. Gallagher, D. Jensen, and B. N. Levine, "MaxProp: routing for vehicle-based disruption-tolerant networks," Proc. $25^{\text {th }}$ IEEE International Conference on Computer Communications, IEEE Press, April. 2006, pp. 1688-1698, doi: 10.1109/INFOCOM.2006.228.

[13] Q. Yang, A. Lim, S. Li, J. Fang, and P. Agrawal, "ACAR: adaptive connectivity aware routing protocol for vehicular ad hoc networks," Proc. 17th International Conference on Computer Communications and Networks, IEEE Press, Aug. 2008, pp. 535-540, doi: 10.1109/ICCCN.2008.ECP.107.

[14] S. A. Rao, M. Pai M.M, M. Boussedjra, and J. Mouzna, "GPSR-L: greedy perimeter stateless routing with lifetime for VANETS," Proc. 8th International Conference on Intelligent Transport System Telecommunications, IEEE Press, Oct. 2008, pp. 299-394, doi: 10.1109/ITST.2008.4740275. 